\title{
SUBDYNAMICS THROUGH TIME SCALES AND SCATTERING MAPS IN QUANTUM FIELD THEORY
}

\author{
Ludovico Lanz $^{(1)}$, Olaf Melsheimer ${ }^{(2)}$ and Bassano Vacchini(1) \\ (1) Dipartimento di Fisica dell'Università di Milano, INFN, Sezione di Milano, \\ Via Celoria 16, I-20133, Milano, Italy \\ ${ }^{(2)}$ Fachbereich Physik, Philipps-Universität, Renthof 7, D-3550 Marburg, Germany
}

\begin{abstract}
It is argued that the dynamics of an isolated system, due to the concrete procedure by which it is separated from the environment, has a non-Hamiltonian contribution. By a unified quantum field theoretical treatment of typical subdynamics, e.g., hydrodynamics, kinetic theory, master equation for a particle interacting with matter, we look for the structure of this more general dynamics.
\end{abstract}

\section{The concept of physical system}

Quantum mechanics (QM) has non-separability as its most striking feature, i.e., one cannot attribute "properties" to parts of a system and therefore typical problems like the measurement process and EPR situations arise. This feature is so deeply rooted in the mathematical structure of QM that we believe one should not try to make it less stringent, e.g., by attempts like "spontaneous reduction" [1]. We prefer instead to weaken the very concept of physical system: usually the "isolation" of a physical system is taken for granted, while in our opinion the way in which isolation is achieved belongs to the very definition of the system. Any attempt inside QM to obtain the subdynamics for a subsystem enforces the introduction of a suitable time scale in order to break the correlations with the environment; in a completely sharp description of the dynamics of a subsystem the physics of the whole universe would enter. The preparation procedure leading to a system, isolated during a time interval $\left[t_{0}, t_{1}\right]$ and confined in a spatial region $\omega$, covers a time interval $\left[T, t_{0}\right]$, that will be called "preparation time". Due to the confinement the basic space-time symmetries are broken and by suitable boundary conditions "peculiar" properties of the system are introduced. This obviously reduces the universal character of the dynamical description, however an important universal behaviour still remains due to symmetry and locality (or short range character) of effective interactions, whose relevance becomes particularly evident in the quantum field theoretical approach. We thus regard a physical system as a part of the world under control by a suitable preparation, whose local behaviour is explained in terms of locally interacting quantum fields. The choice of these fields depends on the level of description of the system. A large part of physics can be explained in terms of quantum fields related to molecules with a typical time scale of the order $\approx 10^{-13} \mathrm{~s}$; a much more refined description arises if the basic fields are related to nuclei and electrons, then the basic theory would be QED and a much smaller time scale $\approx 10^{-23}$ s could be considered: however such role of QED as basic theory of macrosystems is far from being exploited.

In a sense our viewpoint can appear as opposite to the most widely spread one, which we synthetise as follows: particles are the primary systems, related to non-confined quantum 
fields and to basic symmetries, all other systems are structure of particles; one then tries to obtain a typical macroscopic behaviour in some suitable thermodynamic limit. According to us, on the contrary, macroscopic systems are to be taken as the primary systems, even if in their definition time scales and spatial confinement must be carefully taken into account; the theoretical framework for their description is quantum field theory, locality and quantisation taking the place of the atomistic model. In this context particles are a derived concept. The description of non-equilibrium systems is put in the foreground and at least in principle should be performed taking boundary effects into account; procedures like "continuous" limit should be applied only at the end, if one wants to get rid of boundary effects. This standpoint is closer to thermodynamics and electromagnetism, while the former one originates from classical mechanics. The relevance of macroscopic systems for the foundations of QM is the starting point of Ludwig's axiomatic approach to QM [2]. The insistence on the distinction between these two attitudes is due to the fact that they lead in a natural way to two different formulations of the dynamics. In the first approach one associates a wave function $\psi$ to each system $(\psi(\mathbf{x}, t)$ for one particle, $\ldots, \psi\left(\mathbf{x}_{1}, \mathbf{x}_{2}, \ldots, \mathbf{x}_{N}, t\right)$ for $\mathrm{N}$ particles); obviously if one describes situations like "unpolarised" particles it is appropriate to use a statistical operator, in order to take a lack of control of the experimental specification into account. This aspect becomes increasingly important for large $\mathrm{N}$, so that statistical operators are very useful for macroscopic systems, nevertheless the basic dynamics is given by an evolution operator for the wavefunction $\psi$. On the other hand, starting with a macroscopic system, one is led to assume a statistical operator $\hat{\varrho}_{t}$ as the most appropriate mathematical representation of the preparation procedure until time $t$. The set $\mathcal{K}(\mathcal{H})$ of statistical operators on the Hilbert space $\mathcal{H}$ becomes most important and the space $\mathcal{T}(\mathcal{H})$ of trace-class operators, which is generated in a natural way by $\mathcal{K}(\mathcal{H})[\mathcal{K}(\mathcal{H})$ is the base of the base-norm space $\mathcal{T}(\mathcal{H})$ ], plays a role similar to that of $\mathcal{H}$ in the previous formalism. Correspondingly unitary operators on $\mathcal{H}$ in the first approach are replaced by affine maps of $\mathcal{K}(\mathcal{H})$ in $\mathcal{K}(\mathcal{H})$, i.e. by positive, trace-preserving maps on $\mathcal{T}(\mathcal{H})$. If the system is isolated in the time interval $\left[t_{0}, t_{1}\right]$ the spontaneous repreparations $\hat{\varrho}_{t}, t \in\left[t_{0}, t_{1}\right]$, are related together by $\hat{\varrho}_{t}=\mathcal{M}_{t t^{\prime}} \hat{\varrho}_{t^{\prime}}\left(t \geq t^{\prime}\right)$, where the evolution system $\left\{\mathcal{M}_{t^{\prime \prime} t^{\prime}} t^{\prime \prime} \geq t^{\prime}\right\}$ satisfies the composition rule $\mathcal{M}_{t^{\prime \prime \prime} t^{\prime}}=\mathcal{M}_{t^{\prime \prime \prime} t^{\prime \prime}} \mathcal{M}_{t^{\prime \prime} t^{\prime}}\left(t^{\prime} \leq t^{\prime \prime} \leq t^{\prime \prime \prime}\right)$. We stress the fact that there is no reason to assume that $\mathcal{M}_{t^{\prime \prime} t^{\prime}}$ has an inverse. If $\mathcal{M}_{t^{\prime \prime} t^{\prime}}^{-1}$ exists then $\mathcal{M}_{t^{\prime \prime} t^{\prime}}=\hat{U}_{t^{\prime \prime} t^{\prime}} \cdot \hat{U}_{t^{\prime \prime} t^{\prime}}^{\dagger}$ (see for example [3]), with $\hat{U}_{t^{\prime \prime} t^{\prime}}$ unitary or antiunitary operator and one is brought back to the Hilbert space formalism: $\psi_{t^{\prime \prime}}=\hat{U}_{t^{\prime \prime} t^{\prime}} \psi_{t^{\prime}}$. The dynamics in the present framework is indeed more general and has irreversibility as typical phenomenon.

To determine the maps $\mathcal{M}_{t^{\prime \prime} t^{\prime}}$ a choice of relevant observables is necessary: when a time scale is introduced, only those observables should be considered, whose expectation values do not appreciably vary in a time interval of the order of the time scale. It is thus necessary to work in the Heisenberg picture, i.e. with the adjoint map $\mathcal{M}_{t t_{0}}^{\prime}$, and consider expressions of the form $\mathcal{M}_{t t_{0}}^{\prime} \hat{A}, \hat{A}$ being a relevant observable. For the same system different descriptions can be given by different choices of relevant observables and corresponding time scales: e.g., hydrodynamic or kinetic description of a continuum. Skipping questions of mathematical rigour we can assume the differential equation

$$
\frac{d}{d t} \mathcal{M}_{t t_{0}}^{\prime}=\mathcal{L}_{t}^{\prime} \mathcal{M}_{t t_{0}}^{\prime}
$$


and represent $\mathcal{M}_{t t_{0}}^{\prime}$ in the form $\mathcal{M}_{t t_{0}}^{\prime}=T\left(e^{\int_{t_{0}}^{t} d t^{\prime} \mathcal{L}_{t^{\prime}}^{\prime}}\right)$ in terms of the generator $\mathcal{L}_{t}^{\prime}$. It is well known (rigorously for bounded $\mathcal{L}_{t}^{\prime}$ ) that if $\mathcal{M}_{t^{\prime \prime} t^{\prime}}^{\prime}$ has the additional property of complete positivity $(\mathrm{CP}), \mathcal{L}_{t}^{\prime}$ has the Lindblad structure [4]:

$$
\begin{gathered}
\mathcal{L}_{t}^{\prime} \hat{B}=+\frac{i}{\hbar}\left(\hat{H}_{t} \hat{B}-\hat{B} \hat{H}_{t}\right)-\frac{1}{\hbar}\left(\hat{A}_{t} \hat{B}+\hat{B} \hat{A}_{t}\right)+\frac{1}{\hbar} \sum_{j} \hat{L}_{t j}^{\dagger} \hat{B} \hat{L}_{t j} \\
\hat{H}_{t}=\hat{H}_{t}^{\dagger} \quad \hat{A}_{t}=\frac{1}{2} \sum_{j} \hat{L}_{t j} \hat{L}_{t j}^{\dagger} .
\end{gathered}
$$

In our framework the assumption of CP can appear too restrictive since only a suitable subset of observables is relevant and one expects that a modified concept of CP of $\mathcal{M}_{t^{\prime \prime} t^{\prime}}^{\prime}$ relatively to these observables should be given, leading to a more general structure of $\mathcal{L}_{t}^{\prime}$; we shall return to this point in the sequel.

The more general description of the dynamics that we are considering allows the introduction of the concept of trajectory in quantum theory. In fact in the general formalism of continuous measurement approach [5] one has that an evolution system $\left\{\mathcal{M}_{t^{\prime \prime} t^{\prime}} t^{\prime \prime} \geq t^{\prime}\right\}$ with $\mathcal{L}_{t}$ having the Lindblad structure can be decomposed on a space $Y_{t_{0}}^{t_{1}}$ of trajectories for stochastic variables $\xi(t)$. The concept of trajectory is particularly useful in the field of quantum optics, as is shown by the many interesting applications to be found in the literature. More precisely one can consider Wiener and jump processes related to the operators $\hat{L}_{t j}$ in the sense that the expectations of the increments for example in the case of jump processes are given by [6]:

$$
\left\langle d \xi_{j}(t)\right\rangle=d t \operatorname{Tr}\left(\left(\hat{L}_{t j}^{\dagger} \hat{L}_{t j}\right) \hat{\varrho}(t)\right) .
$$

One can define $\sigma$-algebras $\mathcal{B}\left(Y_{t^{\prime}}^{t^{\prime \prime}}\right)$ of subsets $\Omega_{t^{\prime}}^{t^{\prime \prime}}$ of $Y_{t^{\prime}}^{t^{\prime \prime}}$ and construct operation valued measures $\mathcal{F}_{t^{\prime}}^{t^{\prime \prime}}\left(\Omega_{t^{\prime}}^{t^{\prime \prime}}\right)$ on $\mathcal{B}\left(Y_{t^{\prime}}^{t^{\prime \prime}}\right)$ in such a way that $\mathcal{M}_{t^{\prime \prime} t^{\prime}}=\mathcal{F}\left(Y_{t^{\prime}}^{t^{\prime \prime}}\right)$. Then for any decomposition $Y_{t^{\prime}}^{t^{\prime \prime}}=\cup_{\alpha}\left(\Omega_{t^{\prime} \alpha}^{t^{\prime \prime}}\right)$ with disjoint subsets $\Omega_{t^{\prime} \alpha}^{t^{\prime \prime}}$ one has

$$
\mathcal{M}_{t^{\prime \prime} t^{\prime}}=\sum_{\alpha} \mathcal{F}\left(\Omega_{t^{\prime} \alpha}^{t^{\prime \prime}}\right)
$$

One can therefore claim that the quantum dynamics of the system is compatible with the evolution of classical stochastic variables; typically the probability that the trajectory of these variables for $t^{\prime} \leq t \leq t^{\prime \prime}$ belongs to a subset $\Omega_{t^{\prime} \alpha}^{t^{\prime \prime}}$ is given by $p\left(\Omega_{t^{\prime} \alpha}^{t^{\prime \prime}}\right)=$ $\operatorname{Tr}\left(\mathcal{F}_{t^{\prime}}^{t^{\prime \prime}}\left(\Omega_{t^{\prime} \alpha}^{t^{\prime \prime}}\right) \hat{\varrho}\left(t^{\prime}\right)\right)$. The decomposition of $\mathcal{M}_{t^{\prime \prime} t^{\prime}}$ by operation valued stochastic processes $\mathcal{F}_{t^{\prime}}^{t^{\prime \prime}}$ on a suitable trajectory space $Y_{t^{\prime}}^{t^{\prime \prime}}$ is not unique, i.e. there are many compatible objective "classical" pictures which are consistent with the quantum evolution, a feature that can be linked with a "generalised concept of complementarity". The very possibility of recovering some kind of classical insight into QM is due to the non-Hamiltonian evolution; obviously (1.3) would be inconsistent with $\mathcal{M}_{t^{\prime \prime} t^{\prime}}=\hat{U}_{t^{\prime \prime} t^{\prime}} \cdot \hat{U}_{t^{\prime \prime} t^{\prime}}^{\dagger}$, since for $\hat{\varrho}_{t^{\prime}}=\left|\psi_{t^{\prime}}\right\rangle\left\langle\psi_{t^{\prime}}\right|$ the 1.h.s. of (1.3) is a pure state and the r.h.s. is a mixture.

In the framework we have now presented dynamics is given by $\mathcal{L}_{t}^{\prime}[7]$; while one expects that the Hamiltonian part is fixed by the local interactions, the remaining part is connected to the preparation procedure of the isolated system: it cannot be strictly derived 
from a Hamiltonian theory of a larger system surrounding it (in fact also for this larger system an $\mathcal{L}_{t}^{\prime}$ should be determined). The problem arises to give reasonable criteria for the construction of $\mathcal{L}_{t}^{\prime}$. One can expect that near to equilibrium only the Hamiltonian part $\frac{i}{\hbar}[\hat{H}, \cdot]$ is important, as it is clearly indicated by the great success of equilibrium statistical mechanics; however the non-Hamiltonian part of $\mathcal{L}_{t}^{\prime}$ is relevant for irreversible behaviour and for a full explanation of approach to equilibrium: in fact energy conservation $\mathcal{L}_{t}^{\prime} \hat{H}=0$ (at least on the relevant time scale) grants for the existence of an "eigenstate" of $\mathcal{L}_{t}^{\prime}$ with practically zero eigenvalue, while one expects that the other eigenvalues of $\mathcal{L}_{t}^{\prime}$ have a negative real part.

\section{A microsystem interacting with matter}

We shall take on later the problem of an explicit construction of $\hat{\varrho}(t)$ for a macrosystem $\mathcal{S}_{\mathrm{M}}$. We assume now that $\hat{\varrho}(t)$ is given (e.g., the system is at equilibrium) and consider the problem of describing the new system $\mathcal{S}=\mathcal{S}_{\mathrm{M}}+$ a microsystem, $\mathcal{S}$ still being confined inside a region $\omega$. The Hamiltonian of $\mathcal{S}$ is:

$$
\hat{H}=\hat{H}_{0}+\hat{H}_{\mathrm{M}}+\hat{V} \quad \hat{H}_{0}=\sum_{f} E_{f} \hat{a}_{f}^{\dagger} \hat{a}_{f} \quad\left[\hat{a}_{f}, \hat{a}_{g}^{\dagger}\right]_{\mp}=\delta_{f g}
$$

where $E_{f}$ are the eigenvalues of the operator $-\frac{\hbar^{2}}{2 m} \Delta_{2}$ :

$$
-\frac{\hbar^{2}}{2 m} \Delta_{2} u_{f}(\mathbf{x})=E_{f} u_{f}(\mathbf{x}) \quad u_{f}(\mathbf{x})=0 \quad \mathbf{x} \in \partial \omega
$$

Let us assume for the statistical operator of $\mathcal{S}$ the following structure:

$$
\begin{gathered}
\hat{\varrho}(t)=\sum_{g f} \hat{a}_{g}^{\dagger} \hat{\varrho}_{\mathrm{M}}(t) \hat{a}_{f} \varrho_{g f}(t), \\
\hat{a}_{f} \hat{\varrho}_{\mathrm{M}}=0 .
\end{gathered}
$$

The coefficients $\varrho_{g f}$ build a positive, trace one matrix, which can be considered as the representative of a statistical operator $\hat{\varrho}^{(1)}(t)$ in the Hilbert space $\mathcal{H}^{(1)}$ spanned by the states $u_{f}\left(\varrho_{g f}=\left\langle u_{g}\left|\hat{\varrho}^{(1)}\right| u_{f}\right\rangle\right)$. Equation (2.5) indicates that the system $\mathcal{S}_{\mathrm{M}}$ has charge $\hat{Q}=\sum_{f} \hat{a}_{f}^{\dagger} \hat{a}_{f}$ with value zero, i.e. it does not contain the microsystem. Equation (2.4) represents the fact that $\mathcal{S}_{\mathrm{M}}$ has been perturbed by the additional particle and therefore presents a new dynamical behaviour contained in the coefficients $\varrho_{g f}(t)$ that can be picked out studying the time evolution of the observables $\hat{A}=\sum_{h, k} \hat{a}_{h}^{\dagger} A_{h k} \hat{a}_{k}$; in fact the subdynamics of these observables provides the QM of a one-particle system with Hilbert space $\mathcal{H}^{(1)}$, statistical operator $\hat{\varrho}^{(1)}$ and observables $\hat{A}^{(1)}$, with matrix elements $A_{h k}=\left\langle u_{h} \mid \hat{\mathrm{A}}^{(1)} u_{k}\right\rangle_{\mathcal{H}^{(1)}}$, through the formula

$$
\operatorname{Tr}_{\mathcal{H}}(\hat{A} \hat{\varrho}(t))=\sum_{h k} \operatorname{Tr}_{\mathcal{H}}\left(e^{\frac{i}{\hbar} \hat{H} t} \hat{a}_{h}^{\dagger} \hat{a}_{k} e^{-\frac{i}{\hbar} \hat{H} t} \hat{\varrho}\right) A_{h k}=\operatorname{Tr}_{\mathcal{H}^{(1)}}\left(\hat{A}^{(1)} \hat{\varrho}^{(1)}(t)\right)
$$


One has to study the expression $e^{\frac{i}{\hbar} \hat{H} t} \hat{a}_{h}^{\dagger} \hat{a}_{k} e^{-\frac{i}{\hbar} \hat{H} t}$, exploiting the fact that the expectation values $\langle\hat{A}\rangle_{t}$ are "slowly varying" if the matrix $A_{h k}$ is "quasi-diagonal" (if $A_{h k}=\delta_{h k}, \hat{A}$ is a conserved charge). We give here only a sketchy account of the main points (for details see [8]). It proves useful to use in the Heisenberg picture a formalism in $\mathcal{B}(\mathcal{H})$ reminiscent of usual scattering theory in $\mathcal{H}$, by means of superoperators, typically:

$$
\mathcal{H}^{\prime}=\frac{i}{\hbar}[\hat{H}, \cdot], \quad \mathcal{H}_{0}^{\prime}=\frac{i}{\hbar}\left[\hat{H}_{0}+\hat{H}_{\mathrm{M}}, \cdot\right], \quad \mathcal{V}^{\prime}=\frac{i}{\hbar}[\hat{V}, \cdot] .
$$

In such a context operators of the form $\hat{a}_{h}^{\dagger} \hat{a}_{k}$ are "eigenstates" of $\mathcal{H}_{0}^{\prime}$ with eigenvalues $\frac{i}{\hbar}\left(E_{h}-E_{k}\right)$. Setting $\mathcal{U}^{\prime}(t)=e^{\mathcal{H}^{\prime} t}$ one has:

$$
\begin{aligned}
& \mathcal{U}^{\prime}(t)\left(\hat{a}_{h}^{\dagger} \hat{a}_{k}\right)=\left(\mathcal{U}^{\prime}(t) \hat{a}_{h}^{\dagger}\right)\left(\mathcal{U}^{\prime}(t) \hat{a}_{k}\right) \\
&=\int_{-i \infty+\eta}^{+i \infty+\eta} \frac{d z_{1}}{2 \pi i} e^{z_{1} t}\left(\frac{1}{z_{1}-\mathcal{H}^{\prime}} \hat{a}_{h}^{\dagger}\right) \int_{-i \infty+\eta}^{+i \infty+\eta} \frac{d z_{2}}{2 \pi i} e^{z_{2} t}\left(\frac{1}{z_{2}-\mathcal{H}^{\prime}} \hat{a}_{k}\right) \\
& \frac{1}{z-\mathcal{H}^{\prime}}=\frac{1}{z-\mathcal{H}_{0}^{\prime}}+\frac{1}{z-\mathcal{H}_{0}^{\prime}} \mathcal{T}(z) \frac{1}{z-\mathcal{H}_{0}^{\prime}} \quad \text { where } \quad \mathcal{T}(z) \equiv \mathcal{V}^{\prime}+\mathcal{V}^{\prime} \frac{1}{z-\mathcal{H}^{\prime}} \mathcal{V}^{\prime}
\end{aligned}
$$

$\mathcal{T}(z)$ is reminiscent of the usual T-matrix of scattering theory and plays a central role in this treatment: it will be called "scattering map". The operator $\mathcal{T}(z)$ has poles on the imaginary axis for $z=\frac{i}{\hbar}\left(e_{\alpha}-e_{\beta}\right), e_{\alpha}$ being the eigenvalues of $\hat{H}$. In the calculation of expression (2.7) we shall assume that the function $\mathcal{T}(z)$ for $\operatorname{Re} z \approx \varepsilon$ with $\varepsilon \gg \delta$ ( $\delta$ typical spacing between the poles) is smooth enough, so that the only relevant contribution from the singularities of $\left(z-\mathcal{H}^{\prime}\right)^{-1}$ stems from the singularities of $\left(z-\mathcal{H}_{0}^{\prime}\right)^{-1}$; this smoothness property is linked to the fact that the set of poles of $\left(z-\mathcal{H}^{\prime}\right)^{-1}$ goes over to a continuum if the confinement is removed yielding an analytic function with a cut along the imaginary axis, that can be continued across the cut without singularities if no absorption of the microsystem occurs. More precisely $\mathcal{T}(i y+\varepsilon)$ is considered as practically constant for variations $\Delta y \approx \frac{\hbar}{\tau_{0}}$, where $\tau_{0}$ has to be interpreted as a collision time. Treating expression (2.7) we make use of the inequality

$$
\left|E_{h}-E_{k}\right| \ll \frac{\hbar}{\tau_{0}}
$$

whose physical meaning is that the typical variation time $\tau_{1}$ of the quantities $\langle\hat{A}\rangle_{t}$ is much larger than $\tau_{0}$. One then arrives at the following very perspicuous structure for $\mathcal{U}^{\prime}(t)\left(\hat{a}_{h}^{\dagger} \hat{a}_{k}\right)$ :

$$
\begin{gathered}
\mathcal{U}^{\prime}(t)\left(\hat{a}_{h}^{\dagger} \hat{a}_{k}\right)=\hat{a}_{h}^{\dagger} \hat{a}_{k}+t \mathcal{L}^{\prime}\left(\hat{a}_{h}^{\dagger} \hat{a}_{k}\right) \\
\mathcal{L}^{\prime}\left(\hat{a}_{h}^{\dagger} \hat{a}_{k}\right)=\frac{i}{\hbar}\left[\hat{H}_{\mathrm{eff}}, \hat{a}_{h}^{\dagger} \hat{a}_{k}\right]-\frac{1}{\hbar}\left(\left[\hat{\Gamma}^{(1)}, \hat{a}_{h}^{\dagger}\right] \hat{a}_{k}-\hat{a}_{h}^{\dagger}\left[\hat{\Gamma}^{(1)}, \hat{a}_{k}\right]\right)+\frac{1}{\hbar} \sum_{\lambda} \hat{R}_{h \lambda}^{(1) \dagger} \hat{R}_{k \lambda}^{(1)},
\end{gathered}
$$

where $\hat{H}_{\text {eff }}=\hat{H}_{0}+\hat{V}^{\text {eff }}$ and

$$
\hat{V}^{\mathrm{eff}}=\sum_{g r} \hat{a}_{r}^{\dagger} \hat{V}_{r g}^{\mathrm{eff}} \hat{a}_{g}
$$




$$
\begin{aligned}
& =i \hbar \sum_{\substack{\lambda \lambda^{\prime} \\
g r}} \hat{a}_{r}^{\dagger}|\lambda\rangle\left\langle\lambda\left|\frac{1}{2}\left[\left(\mathcal{T}\left(-\frac{i}{\hbar} E_{r}+\varepsilon\right) \hat{a}_{r}\right) \hat{a}_{g}^{\dagger}+\hat{a}_{r}\left(\mathcal{T}\left(\frac{i}{\hbar} E_{g}+\varepsilon\right) \hat{a}_{g}^{\dagger}\right)\right]\right| \lambda^{\prime}\right\rangle\left\langle\lambda^{\prime}\right| \hat{a}_{g} \\
\hat{\Gamma}^{(1)} & =\sum_{g r} \hat{a}_{r}^{\dagger} \hat{\Gamma}_{r g} \hat{a}_{g} \\
& =i \hbar \sum_{\substack{\lambda \lambda^{\prime} \\
g r}} \hat{a}_{r}^{\dagger}|\lambda\rangle\left\langle\lambda\left|\frac{i}{2}\left[\left(\mathcal{T}\left(-\frac{i}{\hbar} E_{r}+\varepsilon\right) \hat{a}_{r}\right) \hat{a}_{g}^{\dagger}-\hat{a}_{r}\left(\mathcal{T}\left(\frac{i}{\hbar} E_{g}+\varepsilon\right) \hat{a}_{g}^{\dagger}\right)\right]\right| \lambda^{\prime}\right\rangle\left\langle\lambda^{\prime}\right| \hat{a}_{g} \\
\hat{R}_{k \lambda}^{(1)} & =\sqrt{2 \varepsilon \hbar^{3}} \sum_{g \lambda^{\prime}} \frac{\left\langle\lambda\left|\left(\mathcal{T}\left(-\frac{i}{\hbar} E_{g}+\varepsilon\right) \hat{a}_{g}\right) \hat{a}_{k}^{\dagger}\right| \lambda^{\prime}\right\rangle}{E_{g}+E_{\lambda}-E_{k}-E_{\lambda^{\prime}}-i \hbar \varepsilon}\left\langle\lambda^{\prime}\right| \hat{a}_{g}
\end{aligned}
$$

and $|\lambda\rangle$ denotes an eigenvector of $\hat{H}_{\mathrm{M}}$ with eigenvalue $E_{\lambda}$ and of $\hat{H}_{0}$ with eigenvalue zero. Eq.(2.10) shows a typical structure arising in the calculation, which we will also find in the more complex situation examined in $\S 3$, where the form of the different operators is further commented on. Let us observe that $\hat{V}_{r g}^{\text {eff }}$ and $\hat{\Gamma}_{r g}$ are not c-number coefficients, but operators acting in the Fock-space for the macrosystem, as stressed by the hats; they are connected respectively to the self-adjoint and anti-self-adjoint part of what can be considered as an operator valued T-matrix. The last contribution displays the "bilinear structure" of the third term in the r.h.s. of (1.2), connected to irreversibility and CP and not reproducible in the Hilbert space formalism, even resorting to an interaction potential which is not self-adjoint. Within the approximation leading to (2.10) one has $\hat{\Gamma}^{(1)} \approx \frac{1}{2} \sum_{h \lambda} \hat{R}_{h \lambda}^{(1) \dagger} \hat{R}_{h \lambda}^{(1)}$ and therefore $\mathcal{L}^{\prime} \hat{N}=0$. Appealing to (2.6) we may obtain an evolution equation for the matrix elements $\varrho_{f g}$ which is meaningful on a time scale much longer than the correlation time for $\mathcal{S}_{\mathrm{M}}$ :

$$
\frac{d \varrho_{g f}}{d t}=-\frac{i}{\hbar}\left(E_{g}-E_{f}\right) \varrho_{g f}+\frac{1}{\hbar} \sum_{h} \varrho_{g h} \mathrm{Q}_{h f}^{\dagger}+\frac{1}{\hbar} \sum_{k} \mathrm{Q}_{g k} \varrho_{k f}+\frac{1}{\hbar} \sum_{\substack{h k \\ \lambda \xi}}\left(\mathrm{L}_{\lambda \xi}\right)_{g k} \varrho_{k h}\left(\mathrm{~L}_{\lambda \xi}\right)_{f h}^{*}
$$

with

$$
\begin{aligned}
\mathrm{Q}_{k f} & =\hbar \operatorname{Tr}_{\mathcal{H}}\left[\left(\mathcal{T}\left(-\frac{i}{\hbar} E_{k}+\varepsilon\right) \hat{a}_{k}\right) \hat{a}_{f}^{\dagger} \hat{\varrho}_{\mathrm{M}}(t)\right] \\
\left(\mathrm{L}_{\lambda \xi}\right)_{k f} & =\sqrt{2 \varepsilon \hbar^{3} \pi_{\xi}}\left\langle\lambda\left|\left[\left(\mathcal{T}\left(-\frac{i}{\hbar} E_{k}+\varepsilon\right) \hat{a}_{k}\right) \hat{a}_{f}^{\dagger}\right] \frac{1}{E_{k}+E_{\lambda}-E_{f}-H_{\mathrm{M}}-i \hbar \varepsilon}\right| \xi(t)\right\rangle ;
\end{aligned}
$$

$\xi(t)$ is a complete system of eigenvectors of $\hat{\varrho}_{\mathrm{M}}(t),\left(\hat{\varrho}_{\mathrm{M}}(t)=\sum_{\xi(t)} \pi_{\xi(t)}|\xi(t)\rangle\langle\xi(t)|\right)$. To show the connection with $(1.2)$ we introduce in $\mathcal{H}^{(1)}$ the operators $\hat{\mathrm{Q}}^{(1)}, \hat{\mathrm{L}}_{\lambda \xi}^{(1)}$ :

$$
\left\langle k\left|\hat{\mathrm{Q}}^{(1)}\right| f\right\rangle=\mathrm{Q}_{k f} \quad, \quad\left\langle k\left|\hat{\mathrm{L}}_{\lambda \xi}^{(1)}\right| f\right\rangle=\left(\mathrm{L}_{\lambda \xi}\right)_{k f},
$$

thus attaining in the Schrödinger picture the full evolution of $\varrho^{(1)}$, given by the typical Lindblad generator:

$$
\frac{d \hat{\varrho}^{(1)}}{d t}=-\frac{i}{\hbar}\left[\hat{\mathrm{H}}_{\mathrm{eff}}, \hat{\varrho}^{(1)}\right]+\frac{1}{2 \hbar}\left\{\left(\hat{\mathrm{Q}}^{(1)}+\hat{\mathrm{Q}}^{(1) \dagger}\right), \hat{\varrho}^{(1)}\right\}+\frac{1}{\hbar} \sum_{\xi, \lambda} \hat{\mathrm{L}}_{\lambda \xi}^{(1)} \hat{\varrho}^{(1)} \hat{\mathrm{L}}_{\lambda \xi}^{(1)} \dagger
$$


where $\hat{\mathrm{H}}_{\text {eff }}^{(1)}=\hat{\mathrm{H}}_{0}^{(1)}+\frac{i}{2}\left(\hat{\mathrm{Q}}^{(1)}-\hat{\mathrm{Q}}^{(1) \dagger}\right)$. Furthermore according to preservation of trace we have $\hat{\mathbf{Q}}^{(1)}+\hat{\mathrm{Q}}^{(1)} \dagger=-\sum_{\lambda \xi} \hat{\mathrm{L}}_{\lambda \xi}^{(1)} \dagger \hat{\mathrm{L}}_{\lambda \xi}^{(1)}$.

As it is well-known (2.11) is apt to describe very different physical situations. If the last contribution, which we will call "incoherent", may be neglected, at least as a first approximation, eq. 2.11) is equivalent to a Schrödinger equation with a possibly complex potential. In the case of a particle interacting with matter this equation is well-suited to describe a coherent optical behaviour, for example in terms of a refractive index, as it is usually done in neutron optics [9, 10] and recently also in atom optics [11, 12]. In this framework the operator $\hat{Q}^{(1)}$ is to be interpreted as an optical potential, which in our formalism is naturally linked to matrix elements of the T-operator, thus showing the connection between the effective, macroscopic description through an index of refraction and quantities characterising the local interactions. The T-operator may be replaced by phenomenological expressions (for example the Fermi pseudo-potential in the case of neutron optics). This picture is particularly useful to deal with particle interferometry. To see how the last contribution may be linked to an interaction having a measuring character let us introduce the reversible mappings $\mathcal{A}_{t^{\prime \prime} t^{\prime}}=\hat{U}_{t^{\prime \prime} t^{\prime}}^{(1)} \cdot \hat{U}_{t^{\prime \prime} t^{\prime}}^{(1)}$, where $\hat{U}_{t^{\prime \prime} t^{\prime}}^{(1)}=$ $T \exp \left(-\frac{i}{\hbar} \int_{t^{\prime}}^{t^{\prime \prime}} d t\left(\hat{\mathrm{H}}_{0}^{(1)}(t)+i \hat{\mathrm{Q}}^{(1)}(t)\right)\right)$, corresponding to a coherent contractive evolution of the microsystem during the time interval $\left[t^{\prime}, t^{\prime \prime}\right]$, and the CP mappings $\mathcal{L}_{\lambda \xi}=\hat{L}_{\lambda \xi}^{(1)}(t)$. $\hat{\mathrm{L}}_{\lambda \xi}^{(1)}{ }^{\dagger}(t)$, having a measuring character, as it is clear from their very structure, reminiscent of the reduction postulate. The expression of the operators $\hat{L}_{\lambda \xi}^{(1)}$ shows how these mappings may be linked with a transition inside the macrosystem specified by the pair of indexes $\xi, \lambda$, as a result of scattering with the microsystem. These transitions are in general not detectable, but under suitable conditions they could prime real events. The solution of (2.11) may be written in the form:

$$
\hat{\varrho}_{t}=\mathcal{A}_{t t_{0}} \hat{\varrho}_{t_{0}}+\sum_{\lambda_{1} \xi_{1}} \int_{t_{0}}^{t} d t_{1} \mathcal{A}_{t t_{1}} \mathcal{L}_{\lambda_{1} \xi_{1}}\left(t_{1}\right) \mathcal{A}_{t_{1} t_{0}} \hat{\varrho}_{t_{0}}+\ldots
$$

that is a sum over subcollections corresponding to the realization of no event, one event and so on. The set of variables $N_{\lambda \xi}(\tau), \tau \geq t_{0}$, (number of transitions up to time $\tau$ ), define a multicomponent classical stochastic process, and (2.12) corresponds to the decomposition of the evolution map on the space of trajectories for $N_{\lambda \xi}(\tau)$. This is a straightforward generalization of the typical "counting process" considered by Srinivas and Davies [13]. Of course other decompositions in terms of operation valued maps are possible on trajectory spaces related to different observables, as indicated in $\S 1$. When the first term in (2.12) is largely predominant a wavelike description as given by the Schrödinger equation is sufficiently accurate and small disturbances, conveyed by the other terms, play no significant role. In a different physical context however, as would be the case for the brownian motion of a particle interacting with an ideal gas, the interplay between the contractive and the incoherent part has a major role. Being interested in the dynamics far away from the walls the quantum number $h$ corresponds to the momentum variable $\mathbf{p}_{h}$, and supposing that momentum transfers are small (Fokker-Planck approximation) one arrives at an equation describing diffusion in phase-space which has the following form 
(see $[14)$ :

$$
\frac{d \hat{\varrho}}{d t}=-\frac{i}{\hbar}\left[\hat{\mathrm{H}}_{\mathrm{eff}}, \hat{\varrho}\right]-D_{p p}[\hat{\mathrm{x}},[\hat{\mathrm{x}}, \hat{\varrho}]]-D_{q q}[\hat{\mathrm{p}},[\hat{\mathrm{p}}, \hat{\varrho}]]-\frac{i \eta}{2 M}[\hat{\mathrm{x}},\{\hat{\mathrm{p}}, \hat{\varrho}\}]
$$

$D_{q q}, D_{q q}$ and $\eta$ being diffusion coefficients linked in different ways to the operators $\hat{\mathrm{L}}_{\lambda \xi}^{(1)}$ and $M$ the mass of the particle.

\section{Theory of a macrosystem: thermodynamic evolu- tion by a scattering map}

We consider a very schematic model of macrosystem in the non-relativistic case built by one type of molecules with mass $m$ confined inside a region $\omega$, interacting by a two body potential $V(|\mathbf{x}-\mathbf{y}|)$; for the sake of simplicity no internal structure of the molecules is taken into account. In the field theoretical language the system is described by a quantum Schrödinger field (QSF):

$$
\begin{gathered}
\hat{\psi}(\mathbf{x})=\sum_{f} u_{f}(\mathbf{x}) \hat{a}_{f} \quad\left[\hat{a}_{f}, \hat{a}_{g}^{\dagger}\right]_{ \pm}=\delta_{f g} \\
-\frac{\hbar^{2}}{2 m} \Delta_{2} u_{f}(\mathbf{x})=E_{f} u_{f}(\mathbf{x}), \quad u_{f}(\mathbf{x})=0 \quad \mathbf{x} \in \partial \omega .
\end{gathered}
$$

We shall assume the following Hamiltonian to take local interactions and confinement into account:

$$
\begin{gathered}
\hat{H}=\sum_{f} E_{f} \hat{a}_{f}^{\dagger} \hat{a}_{f}+\frac{1}{2} \sum_{\substack{l_{1} l_{2} \\
f_{1} f_{2}}} \hat{a}_{l_{1}}^{\dagger} \hat{a}_{l_{2}}^{\dagger} V_{l_{1} l_{2} f_{2} f_{1}} \hat{a}_{f_{2}} \hat{a}_{f_{1}} \\
V_{l_{1} l_{2} f_{2} f_{1}}=\int_{\omega} d^{3} \mathbf{x} \int_{\omega} d^{3} \mathbf{y} u_{l_{1}}^{*}(\mathbf{x}) u_{l_{2}}^{*}(\mathbf{y}) V(|\mathbf{x}-\mathbf{y}|) u_{f_{2}}(\mathbf{y}) u_{f_{1}}(\mathbf{x}) .
\end{gathered}
$$

Eq.(3.1) is linked to the basic "local" Hamiltonian for the non-confined field (NC)

$$
\begin{aligned}
& \hat{H}_{\mathrm{NC}}= \int d^{3} \mathbf{x} \frac{\hbar^{2}}{2 m} \operatorname{grad} \hat{\psi}_{\mathrm{NC}}^{\dagger}(\mathbf{x}) \cdot \operatorname{grad} \hat{\psi}_{\mathrm{NC}}(\mathbf{x}) \\
&+\frac{1}{2} \int d^{3} \mathbf{x} d^{3} \mathbf{r} \hat{\psi}_{\mathrm{NC}}^{\dagger}\left(\mathbf{x}-\frac{\mathbf{r}}{2}\right) \hat{\psi}_{\mathrm{NC}}^{\dagger}\left(\mathbf{x}+\frac{\mathbf{r}}{2}\right) V(r) \hat{\psi}_{\mathrm{NC}}\left(\mathbf{x}+\frac{\mathbf{r}}{2}\right) \hat{\psi}_{\mathrm{NC}}\left(\mathbf{x}-\frac{\mathbf{r}}{2}\right) \\
& {\left[\hat{\psi}_{\mathrm{NC}}(\mathbf{x}), \hat{\psi}_{\mathrm{NC}}^{\dagger}\left(\mathbf{x}^{\prime}\right)\right]_{ \pm}=\delta\left(\mathbf{x}-\mathbf{x}^{\prime}\right), }
\end{aligned}
$$

simply selecting the part of $\hat{H}_{\mathrm{NC}}$ related to the "normal modes" $u_{f}$ typical of the confinement: in fact the preparation procedure should imply a kind of relaxation of $\hat{\psi}_{\mathrm{NC}}(\mathbf{x})$ to $\hat{\psi}(\mathbf{x})$. Skipping this problem and also the related question of the full explicit structure of $\mathcal{L}^{\prime}$ for a realistic system, we shall simply take the Hamiltonian (3.2) containing only the normal modes of the field inside $\omega$. If we are interested in a hydrodynamic description 
relevant observables are constructed starting with the densities of the typical constants of motion, mass and energy:

$$
\begin{aligned}
\hat{\rho}_{m}(\mathbf{x})= & m \hat{\psi}^{\dagger}(\mathbf{x}) \hat{\psi}(\mathbf{x}) \\
\hat{e}(\mathbf{x})= & \frac{\hbar^{2}}{2 m} \operatorname{grad} \hat{\psi}^{\dagger}(\mathbf{x}) \cdot \operatorname{grad} \hat{\psi}(\mathbf{x}) \\
& +\frac{1}{2} \int_{\omega_{x}} d^{3} \mathbf{r} \hat{\psi}^{\dagger}\left(\mathbf{x}-\frac{\mathbf{r}}{2}\right) \hat{\psi}^{\dagger}\left(\mathbf{x}+\frac{\mathbf{r}}{2}\right) V(r) \hat{\psi}\left(\mathbf{x}+\frac{\mathbf{r}}{2}\right) \hat{\psi}\left(\mathbf{x}-\frac{\mathbf{r}}{2}\right),
\end{aligned}
$$

where the dependence of $\omega_{x}$ on $\mathbf{x}$ is generally negligible if $V(r)$ is a short range potential. In the case of a kinetic description we replace (3.4) by the "Boltzmann" operator density $\hat{f}(\mathbf{x}, \mathbf{p})=m \sum_{h k} \hat{a}_{h}^{\dagger}\left\langle u_{h}\left|\hat{\mathbf{F}}^{(1)}(\mathbf{x}, \mathbf{p})\right| u_{k}\right\rangle \hat{a}_{k}$, where $\hat{\mathbf{F}}^{(1)}$ is the density of joint one particle position-momentum observables [15, 16]. These densities lead to slowly varying quantities if they are integrated over regions large enough in space or phase-space, since one has constants if the integration is extended over the whole space. The constants of motion leading to this subdynamics are linked to very fundamental symmetries: time translation invariance and gauge symmetry. Our relevant observables have the general structure:

$$
\begin{gathered}
\sum_{h k} \hat{a}_{h}^{\dagger} A_{h k}(\xi) \hat{a}_{k} \quad, \quad \sum_{\substack{k_{1} k_{2} \\
h_{1} h_{2}}} \hat{a}_{h_{1}}^{\dagger} \hat{a}_{h_{2}}^{\dagger} A_{h_{1} h_{2} k_{2} k_{1}}(\mathbf{x}) \hat{a}_{k_{2}} \hat{a}_{k_{1}} \\
A_{h_{1} h_{2} k_{2} k_{1}}(\mathbf{x})=\frac{1}{2} \int_{\omega_{x}} d^{3} \mathbf{r} u_{h_{1}}^{*}\left(\mathbf{x}-\frac{\mathbf{r}}{2}\right) u_{h_{2}}^{*}\left(\mathbf{x}+\frac{\mathbf{r}}{2}\right) V(r) u_{k_{2}}\left(\mathbf{x}+\frac{\mathbf{r}}{2}\right) u_{k_{1}}\left(\mathbf{x}-\frac{\mathbf{r}}{2}\right) .
\end{gathered}
$$

We thus have to study in Heisenberg picture the expressions:

$$
\sum_{h k} e^{\frac{i}{\hbar} \hat{H} t} \hat{a}_{h}^{\dagger} \hat{a}_{k} e^{-\frac{i}{\hbar} \hat{H} t} A_{h k}(\xi), \quad \sum_{\substack{h_{1} h_{2} \\ k_{1} k_{2}}} e^{\frac{i}{\hbar} \hat{H} t} \hat{a}_{h_{1}}^{\dagger} \hat{a}_{h_{2}}^{\dagger} \hat{a}_{k_{2}} \hat{a}_{k_{1}} e^{-\frac{i}{\hbar} \hat{H} t} A_{h_{1} h_{2} k_{2} k_{1}}(\mathbf{x}),
$$

and shall take into account that by the slow variability only terms that are "diagonal enough" are really relevant; the sums should be restricted to indexes such that:

$$
\frac{1}{\hbar}\left|E_{h}-E_{k}\right|<\frac{1}{\tau_{1}} \quad \frac{1}{\hbar}\left|E_{h_{1}}+E_{h_{2}}-E_{k_{1}}-E_{k_{2}}\right|<\frac{1}{\tau_{1}},
$$

where $\tau_{1}$ is the characteristic variation time of the relevant quantities. We would like to stress the fact that the QSF is the basic tool to describe a massive continuum, just like the quantum electromagnetic field describes a massless continuum. The dynamics of the QSF, $\hat{\psi}(\mathbf{x}, t)=e^{\frac{i}{\hbar} \hat{H} t} \hat{\psi}(\mathbf{x}) e^{-\frac{i}{\hbar} \hat{H} t}$, in terms of which one can rewrite (3.6), is given by the simple field equation

$$
i \hbar \frac{\partial}{\partial t} \hat{\psi}(\mathbf{x}, t)=-\frac{\hbar^{2}}{2 m} \Delta_{2} \hat{\psi}(\mathbf{x}, t)+\int d^{3} \mathbf{y} \hat{\psi}^{\dagger}(\mathbf{y}, t) V(|\mathbf{x}-\mathbf{y}|) \hat{\psi}(\mathbf{y}, t) \hat{\psi}(\mathbf{x}, t),
$$

however no such equation holds for the expectation value of the field $\psi(\mathbf{x}, t)=\langle\hat{\psi}(\mathbf{x}, t)\rangle$ due to correlations in the non-linear term; $\psi(\mathbf{x}, t)$ is not useful to calculate the expectations of operators (3.6) and therefore a classical Schrödinger field equation for $\psi(\mathbf{x}, t)$ has no 
physical meaning in general. In this respect the case of electromagnetism, where no selfinteraction of the field occurs, is deeply different and allows classical electrodynamics to play an important role. To the macrosystem one associates typical "thermodynamic state" parameters: the velocity field $\mathbf{v}(\mathbf{x}, t)$, the temperature field $\beta(\mathbf{x}, t)$, the chemical potential field $\mu(\mathbf{x}, t)$ in the case of the hydrodynamic description or more generally a field $\mu(\mathbf{x}, \mathbf{p}, t)$ on the one-particle phase-space in the kinetic case [17]. The parameters $\beta(\mathbf{x}, t)$ and $\mu(\mathbf{x}, t)(\mu(\mathbf{x}, \mathbf{p}, t))$ determine the expectation values of energy density and mass density (the Boltzmann operator); let us briefly recall how the relation between state variables and expectation values is established [18]. At any time $t$ one considers the whole set of statistical operators $\{\hat{w}\}$ which yield the expectation values assigned at that time:

$$
\begin{aligned}
&\left\langle\hat{e}^{(0)}(\mathbf{x})\right\rangle_{t}=\operatorname{Tr}\left(\hat{e}^{(0)}(\mathbf{x}) \hat{w}\right),\left\langle\hat{\rho}_{m}(\mathbf{x})\right\rangle_{t}=\operatorname{Tr}\left(\hat{\rho}_{m}(\mathbf{x}) \hat{w}\right),\left\langle\hat{f}^{(0)}(\mathbf{x}, \mathbf{p})\right\rangle_{t}=\operatorname{Tr}\left(\hat{f}^{(0)}(\mathbf{x}, \mathbf{p}) \hat{w}\right) \\
& \hat{e}^{(0)}(\mathbf{x})= \frac{1}{2 m}\left(i \hbar \frac{\partial}{\partial \mathbf{x}}-m \mathbf{v}(\mathbf{x}, t)\right) \hat{\psi}^{\dagger}(\mathbf{x}) \cdot\left(-i \hbar \frac{\partial}{\partial \mathbf{x}}-m \mathbf{v}(\mathbf{x}, t)\right) \hat{\psi}(\mathbf{x}) \\
&+\frac{1}{2} \int_{\omega_{x}} d \mathbf{r} \hat{\psi}^{\dagger}\left(\mathbf{x}-\frac{\mathbf{r}}{2}\right) \hat{\psi}^{\dagger}\left(\mathbf{x}+\frac{\mathbf{r}}{2}\right) V(r) \hat{\psi}\left(\mathbf{x}+\frac{\mathbf{r}}{2}\right) \hat{\psi}\left(\mathbf{x}-\frac{\mathbf{r}}{2}\right) \\
& \hat{\rho}_{m}(\mathbf{x})= \hat{\rho}_{m}^{(0)}(\mathbf{x})=m \hat{\psi}^{\dagger}(\mathbf{x}) \hat{\psi}(\mathbf{x}), \quad \hat{f}^{(0)}(\mathbf{x}, \mathbf{p})=\hat{f}(\mathbf{x}, \mathbf{p}-m \mathbf{v}(\mathbf{x}, t))
\end{aligned}
$$

where the quantities indexed by (0) (depending explicitly on the velocity field $\mathbf{v}(\mathbf{x}, t)$ ) represent densities in the reference frame in which the continuum is locally at rest. The velocity field is related to the expectation value of the momentum density $\hat{\mathbf{p}}(\mathbf{x})$ through the relation $\left\langle\hat{\mathbf{p}}^{(0)}(\mathbf{x})\right\rangle=0$, where

$$
\hat{\mathbf{p}}^{(0)}(\mathbf{x})=\frac{1}{2}\left\{\hat{\psi}^{\dagger}(\mathbf{x})\left(-i \hbar \frac{\partial}{\partial \mathbf{x}}-m \mathbf{v}(\mathbf{x}, t)\right) \hat{\psi}(\mathbf{x})+\left[\left(i \hbar \frac{\partial}{\partial \mathbf{x}}-m \mathbf{v}(\mathbf{x}, t)\right) \hat{\psi}^{\dagger}(\mathbf{x})\right] \hat{\psi}(\mathbf{x})\right\}
$$

or equivalently $\langle\hat{\mathbf{p}}(\mathbf{x})\rangle_{t}=\mathbf{v}(\mathbf{x}, t)\left\langle\hat{\rho}_{m}(\mathbf{x})\right\rangle_{t}$. Then one looks for a statistical operator in the set $\{\hat{w}\}$ such that the Von-Neumann entropy $S=-k \operatorname{Tr}(\hat{w} \log \hat{w})$ is maximal, i.e. the most unbiased choice of a statistical operator leading to the given expectation values. The unique solution of this problem is

$$
\hat{w}[\beta(t), \mu(t), \mathbf{v}(t)]=\frac{e^{-\int_{\omega} d^{3} \mathbf{x} \beta(\mathbf{x}, t)\left[\hat{e}^{(0)}(\mathbf{x})-\mu(\mathbf{x}, t) \hat{\rho}_{m}(\mathbf{x})\right]}}{\operatorname{Tr} e^{-\int_{\omega} d^{3} \beta \beta(\mathbf{x}, t)\left[\hat{e}^{(0)}(\mathbf{x})-\mu(\mathbf{x}, t) \hat{\rho}_{m}(\mathbf{x})\right]}}
$$

and analogously in the kinetic case.

The corresponding $S=-k \operatorname{Tr}(\hat{w}[\beta(t), \mu(t), \mathbf{v}(t)] \log \hat{w}[\beta(t), \mu(t), \mathbf{v}(t)])$ is the thermodynamic entropy of the macrosystem. If the time evolution of the expectation values $\left\langle\hat{e}^{(0)}(\mathbf{x})\right\rangle_{t},\left\langle\hat{\rho}_{m}(\mathbf{x})\right\rangle_{t},\left(\langle\hat{f}(\mathbf{x}, \mathbf{p})\rangle_{t}\right)$ is given by the Hamiltonian evolution (3.6) or more generally by a map $\mathcal{M}_{t t_{0}}^{\prime}$, having a preadjoint $\mathcal{M}_{t t_{0}}$ which does not decrease the Von-Neumann entropy, one immediately has that the thermodynamic entropy is non-decreasing. In this way one establishes the second principle of thermodynamics on a very clear dynamical basis. 
In the simplest scheme of macroscopic dynamics the thermodynamic state parameters $\mathbf{v}(\mathbf{x}, t), \beta(\mathbf{x}, t), \mu(\mathbf{x}, t)(\mu(\mathbf{x}, \mathbf{p}, t))$ at time $t_{0}$ determine its evolution for $t>t_{0}$, e.g., by differential equations. Phenomenology shows that this is very often the case. Tackling the problem from the theoretical viewpoint one is induced, considering the operators

$$
\begin{array}{rlrlrl}
\dot{\hat{\rho}}_{m}(\mathbf{x}) & =\frac{i}{\hbar}\left[\hat{H}, \hat{\rho}_{m}(\mathbf{x})\right] & \dot{\hat{\mathbf{p}}}(\mathbf{x}) & = & \frac{i}{\hbar}[\hat{H}, \hat{\mathbf{p}}(\mathbf{x})] \\
\dot{\hat{e}}(\mathbf{x}) & =\frac{i}{\hbar}[\hat{H}, \hat{e}(\mathbf{x})] & (\hat{\hat{f}}(\mathbf{x}, \mathbf{p}) & \left.=\frac{i}{\hbar}[\hat{H}, \hat{f}(\mathbf{x}, \mathbf{p})]\right),
\end{array}
$$

to calculate their expectations with the statistical operator given by (3.9). This leads to wrong results as can be seen from the fact that the expectation values of the currents which can be associated, through a conservation equation, to these operators would vanish [19], due to time reversal invariance of microphysics, thus failing to describe any dissipative flow (e.g., heat conduction, viscosity, etc.). The idea of a time scale for the thermodynamic evolution and of a related subdynamics for the basic densities leads to a refinement of the aforementioned procedure: assume that $\frac{i}{\hbar}[\hat{H}, \cdot \cdot]$ can be replaced by a mapping $\mathcal{L}^{\prime}$, initially defined on the linearly independent elements $\hat{a}_{h}^{\dagger} \hat{a}_{k}, \hat{a}_{h_{1}}^{\dagger} \hat{a}_{h_{2}}^{\dagger} \hat{a}_{k_{2}} \hat{a}_{k_{1}}$, giving the slow time evolution of the relevant variables. In this way not only the statistical operator $\hat{w}[\beta(t), \mu(t), \mathbf{v}(t)]$, but also the evolution operator is tuned to the relevant observables. Then one has the following set of closed evolution equations for the thermodynamic fields $\mathbf{v}(\mathbf{x}, t), \beta(\mathbf{x}, t), \mu(\mathbf{x}, t),(\mu(\mathbf{x}, \mathbf{p}, t))$ related to the basic observables $\hat{A}=\hat{\rho}_{m}(\mathbf{x}), \hat{\mathbf{p}}(\mathbf{x}), \hat{e}(\mathbf{x}),(\hat{f}(\mathbf{x}, \mathbf{p}))$ :

$$
\frac{d}{d t} \operatorname{Tr}(\hat{A} \hat{w}[\beta(t), \mu(t), \mathbf{v}(t)])=\operatorname{Tr}\left(\left(\mathcal{L}^{\prime} \hat{A}\right) \hat{w}[\beta(t), \mu(t), \mathbf{v}(t)]\right) .
$$

The non-Hamiltonian form of the map $\mathcal{L}^{\prime}$ eliminates the aforementioned difficulties with vanishing dissipative flows; preliminary investigations of the consequences of (3.10) in the case of a dilute gas indicate that it could be the right solution. The map $\mathcal{L}^{\prime}$ that adequately replaces $\frac{i}{\hbar}[\hat{H}, \cdot]$ for the slow variables must generate an evolution of the relevant observables that preserves their positivity properties (e.g., $\left.\hat{\rho}_{m}(\mathbf{x}), \hat{f}(\mathbf{x}, \mathbf{p})\right)$ and also conservation of mass $\left(\hat{M}=\int d^{3} \mathbf{x} \hat{\rho}_{m}(\mathbf{x})=\int d^{3} \mathbf{x} d^{3} \mathbf{p} \hat{f}(\mathbf{x}, \mathbf{p})\right)$ and of energy $\left(\hat{E}=\int d^{3} \mathbf{x} \hat{e}(\mathbf{x})\right)$. Then $\mathcal{L}^{\prime} \hat{M}=0$ and $\mathcal{L}^{\prime} \hat{E}=0$, while positivity with respect to observables constructed in terms of creation and annihilation operators could arise by a stronger property, reminding CP:

$$
\sum_{h k}\left\langle\psi_{h} \mid \mathcal{U}^{\prime}\left(\hat{a}_{h}^{\dagger} \hat{a}_{k}\right) \psi_{k}\right\rangle>0 \quad \sum_{\substack{h_{1} h_{2} \\ k_{1} k_{2}}}\left\langle\psi_{h_{1} h_{2}} \mid \mathcal{U}^{\prime}\left(\hat{a}_{h_{1}}^{\dagger} \hat{a}_{h_{2}}^{\dagger} \hat{a}_{k_{2}} \hat{a}_{k_{1}}\right) \psi_{k_{1} k_{2}}\right\rangle>0
$$

for any choice of $\left\{\psi_{h}\right\}$ and $\left\{\psi_{h_{1} h_{2}}\right\}$.

The time evolution of the typical expressions (3.6) can be studied by a procedure quite similar to that already shown in $\S 2$, based on the representation (2.7) in terms of the "superoperator" $\mathcal{H}_{0}$, having $\hat{a}_{h}^{\dagger} \hat{a}_{k}, \hat{a}_{h_{1}}^{\dagger} \hat{a}_{h_{2}}^{\dagger} \hat{a}_{k_{2}} \hat{a}_{k_{1}}$ as eigenstates and of the superoperator $\mathcal{T}(z)$, which was called scattering map. If suitable smoothness properties of $\mathcal{T}(z)$ occur, essentially only the poles of $\left(z-\mathcal{H}_{0}^{\prime}\right)^{-1}$ contribute to the calculation of (2.7), so that the following asymptotic representation holds:

$$
\mathcal{U}^{\prime}(t)\left(\hat{a}_{h}^{\dagger} \hat{a}_{k}\right)=\hat{a}_{h}^{\dagger} \hat{a}_{k}+t \mathcal{L}^{\prime}\left(\hat{a}_{h}^{\dagger} \hat{a}_{k}\right) \quad \tau_{0} \ll t \ll \frac{\hbar}{\left|E_{h}-E_{k}\right|} ;
$$


$\tau_{0}$ is linked to smoothness properties of $\mathcal{T}(z)$ and can be interpreted as the typical duration of a collision between two particles interacting through the potential $V(|\mathbf{x}-\mathbf{y}|) ; \tau_{0}$ fixes a time scale that is assumed to be much smaller than the typical variation time $\tau_{1}$ of our relevant observables. $\mathcal{L}^{\prime}$ is a linear mapping defined initially on the linearly independent elements $\hat{a}_{h}^{\dagger} \hat{a}_{k}, \hat{a}_{h_{1}}^{\dagger} \hat{a}_{h_{2}}^{\dagger} \hat{a}_{k_{2}} \hat{a}_{k_{1}}$. For brevity we simply describe the structure of $\mathcal{L}^{\prime}$, skipping the derivation. The formalism produces the typical structure of two-particle QM, with an N-body correction due to the Pauli principle. A two-particle scattering operator is defined by

$$
\hat{\mathbf{T}}^{(2)}(z)=\hat{V}^{(2)}+\hat{V}^{(2)} \frac{1}{z-\hat{H}_{L}^{(2)}} \hat{V}_{L}^{(2)} \quad \hat{H}_{L}^{(2)}=\hat{H}_{0}^{(2)}+\hat{V}_{L}^{(2)},
$$

where these operators, labelled by the index (2), are defined in the Hilbert space $\mathcal{H}^{(2)}$ of two identical particles by matrix elements in the two-particle (symmetric or antisymmetric) basis $\left|l_{2} l_{1}\right\rangle$; the matrix elements are:

$$
\begin{aligned}
\left\langle l_{2} l_{1}\left|\hat{H}_{0}^{(2)}\right| f_{2} f_{1}\right\rangle & =\left(E_{f_{1}}+E_{f_{2}}\right) \frac{1}{2 !}\left(\delta_{l_{2} f_{2}} \delta_{l_{1} f_{1}} \pm \delta_{l_{2} f_{1}} \delta_{l_{1} f_{2}}\right) \\
\left\langle l_{2} l_{1}\left|\hat{V}^{(2)}\right| f_{2} f_{1}\right\rangle & =V_{l_{1} l_{2} f_{2} f_{1}} \\
\left\langle l_{2} l_{1}\left|\hat{V}_{L}^{(2)}\right| f_{2} f_{1}\right\rangle & =\left(1 \pm \hat{n}_{l_{1}} \pm \hat{n}_{l_{2}}\right) V_{l_{1} l_{2} f_{2} f_{1}}
\end{aligned}
$$

the coefficients $E_{f}, V_{l_{1} l_{2} f_{2} f_{1}}$ are given in (3.2) and (3.3), the factor $\left(1 \pm \hat{n}_{l_{1}} \pm \hat{n}_{l_{2}}\right)$ is given in a more indirect way: the "two-particle" QM expressed by the aforementioned operators provides c-number coefficients in Fock-space operator expressions initially defined on the Fock-space basis $\left|\ldots n_{f} \ldots\right\rangle, n_{f}$ being the occupation numbers of the different field modes $f: n_{f} \in \mathrm{N}$ in the Bose case, $n_{f}=0,1$ in the Fermi case; therefore the factor $\left(1 \pm \hat{n}_{l_{1}} \pm \hat{n}_{l_{2}}\right)$ depends on the Fock-space basis elements on which the final Fock-space operator is acting. Also the adjoint operator in the two-particle Hilbert space will be useful:

$$
\hat{V}_{R}^{(2)}=\hat{V}_{L}^{(2) \dagger}, \quad \hat{H}_{R}^{(2)}=\hat{H}_{L}^{(2) \dagger}, \quad\left[\hat{\mathbf{T}}^{(2)}(z)\right]^{\dagger}=\hat{V}^{(2)}+\hat{V}_{R}^{(2)} \frac{1}{z^{*}-\hat{H}_{R}^{(2)}} \hat{V}^{(2)} .
$$

The superoperator $\mathcal{L}^{\prime}$ consists of an Hamiltonian part $\frac{i}{\hbar}\left[\hat{H}_{\text {eff }}, \cdot\right]$ and of a part, analogous to the one in (2.10), reminding the Lindblad structure (1.2). The formally self-adjoint Hamilton operator $\hat{H}_{\mathrm{eff}}$ is initially defined on the Fock-space basis $\left|\ldots n_{f} \ldots\right\rangle$ by the following expression:

$$
\begin{aligned}
\hat{H}_{\mathrm{eff}} & =\sum_{f} E_{f} \hat{a}_{f}^{\dagger} \hat{a}_{f}+\frac{1}{2} \sum_{\substack{l_{1} l_{2} \\
f_{1} f_{2}}} \hat{a}_{l_{1}}^{\dagger} \hat{a}_{l_{2}}^{\dagger} V_{l_{1} l_{2} f_{2} f_{1}}^{e f f} \hat{a}_{f_{2}} \hat{a}_{f_{1}} \\
V_{l_{1} l_{2} f_{2} f_{1}}^{e f f} & =\left\langle l_{2} l_{1}\left|\frac{1}{2}\left(\hat{\mathbf{T}}^{(2)}\left(E_{f_{1}}+E_{f_{2}}+i \hbar \varepsilon\right)+\left[\hat{\mathbf{T}}^{(2)}\left(E_{l_{1}}+E_{l_{2}}+i \hbar \varepsilon\right)\right]^{\dagger}\right)\right| f_{2} f_{1}\right\rangle .
\end{aligned}
$$

By comparison with (3.1) one can notice that introducing the time scale $\tau \gg \tau_{0}$ the coefficients $V_{l_{1} l_{2} f_{2} f_{1}}$ related to the basic interaction between the field modes is replaced by $V_{l_{1} l_{2} f_{2} f_{1}}^{e f f}$, linked with a full, Pauli principle corrected description of the two body collisions in the medium, expressed in terms of the self-adjoint part of the operator $\hat{\mathbf{T}}^{(2)}(z)$. The antiself-adjoint part $\frac{i}{2}\left(\hat{\mathbf{T}}^{(2)}(z)-\hat{\mathbf{T}}^{(2)}(z)^{\dagger}\right)$ is not zero if one goes beyond Born approximation 
and provides a contribution to $\mathcal{L}^{\prime}$ analogous to the second term in the l.h.s. of (1.2), of the form $-\frac{1}{\hbar}\left(\left[\hat{\Gamma}^{(2)}, \hat{a}_{h}^{\dagger}\right] \hat{a}_{k}-\hat{a}_{h}^{\dagger}\left[\hat{\Gamma}^{(2)}, \hat{a}_{k}\right]\right)$, that due to sign "-" cannot be rewritten as $\left[\hat{\Gamma}^{(2)}, \cdot\right]$; the operator $\hat{\Gamma}^{(2)}$ is defined on the Fock-space basis by

$$
\frac{1}{2} \sum_{\substack{f_{1} f_{2} \\ l_{1} l_{2}}} \hat{a}_{l_{1}}^{\dagger} \hat{a}_{l_{2}}^{\dagger}\left\langle l_{2} l_{1}\left|\frac{i}{2}\left(\hat{\mathbf{T}}^{(2)}\left(E_{f_{1}}+E_{f_{2}}+i \hbar \varepsilon\right)-\left[\hat{\mathbf{T}}^{(2)}\left(E_{l_{1}}+E_{l_{2}}+i \hbar \varepsilon\right)\right]^{\dagger}\right)\right| f_{2} f_{1}\right\rangle \hat{a}_{f_{2}} \hat{a}_{f_{1}} .
$$

At this point one immediately expects a third contribution to $\mathcal{L}^{\prime}$ related to the product structure $\hat{a}_{h}^{\dagger} \hat{a}_{k}$ and involving both $\hat{\mathbf{T}}^{(2)}$ and $\hat{\mathbf{T}}^{(2)^{\dagger}}$; this contribution is given by $\frac{1}{\hbar} \sum_{\lambda} \hat{R}_{h \lambda}^{(2) \dagger} \hat{R}_{k \lambda}^{(2)}$ and reminds the structure of the third term at the l.h.s. of the Lindblad expression (1.2), where it accounted for decoherence (or state reduction, or event production). The operators $\hat{R}_{k \lambda}^{(2)}$ are defined on the Fock-space basis by:

$$
\hat{R}_{k \lambda}^{(2)}=-i \sqrt{2 \varepsilon\left(1 \pm \hat{n}_{\lambda} \pm \hat{n}_{k}\right)} \sum_{f_{1} f_{2}} \frac{\left\langle k \lambda\left|\hat{\mathbf{T}}^{(2)}\left(E_{f_{1}}+E_{f_{2}}+i \hbar \varepsilon\right)\right| f_{2} f_{1}\right\rangle}{E_{k}+E_{\lambda}-E_{f_{1}}-E_{f_{2}}-i \hbar \varepsilon} \hat{a}_{f_{2}} \hat{a}_{f_{1}} .
$$

The factor $\sqrt{2 \varepsilon\left(1 \pm \hat{n}_{\lambda} \pm \hat{n}_{k}\right)}$ arises in the approximate factorisation of a Pauli correction term depending both on $\hat{n}_{k}$ and $\hat{n}_{h}$ :

$$
2 \varepsilon\left(1 \pm \hat{n}_{\lambda} \pm \frac{1}{2}\left(\hat{n}_{h}+\hat{n}_{k}\right)\right) \approx \sqrt{2 \varepsilon\left(1 \pm \hat{n}_{\lambda} \pm \hat{n}_{h}\right)} \sqrt{2 \varepsilon\left(1 \pm \hat{n}_{\lambda} \pm \hat{n}_{k}\right)}
$$

this factorisation, which is a good approximation if the Pauli corrections are not very large, is a typical quantum condition, which together with $\tau_{0} \ll \tau_{1}$ must be satisfied for the validity of the simple thermodynamic behaviour that we are considering in this section. The final structure of $\mathcal{L}^{\prime}$ is formally the same as in (2.10)

$$
\mathcal{L}^{\prime} \hat{a}_{h}^{\dagger} \hat{a}_{k}=\frac{i}{\hbar}\left[\hat{H}_{\mathrm{eff}}, \hat{a}_{h}^{\dagger} \hat{a}_{k}\right]-\frac{1}{\hbar}\left(\left[\hat{\Gamma}^{(2)}, \hat{a}_{h}^{\dagger}\right] \hat{a}_{k}-\hat{a}_{h}^{\dagger}\left[\hat{\Gamma}^{(2)}, \hat{a}_{k}\right]\right)+\frac{1}{\hbar} \sum_{\lambda} \hat{R}_{h \lambda}^{(2) \dagger} \hat{R}_{k \lambda}^{(2)},
$$

the main difference lying in the space in which these operators act, according to the two different physical situations. As a consequence of unitarity of $\mathcal{U}^{\prime}$ one can prove that within the approximation leading to expression (3.19) one has:

$$
\hat{\Gamma}^{(2)} \approx \frac{1}{4} \sum_{h \lambda} \hat{R}_{h \lambda}^{(2) \dagger} \hat{R}_{h \lambda}^{(2)}
$$

therefore one can replace the expression $\hat{\Gamma}^{(2)} \approx \frac{1}{4} \sum_{h \lambda} \hat{R}_{h \lambda}^{(2) \dagger} \hat{R}_{h \lambda}^{(2)}$ in (3.19): in this way the conservation relation $\mathcal{L}^{\prime} \hat{M}=0$ is exactly satisfied. It can be easily shown that $\sum_{h k}\left\langle\psi_{h} \mid\left(\left[1+\tau \mathcal{L}^{\prime}\right] \hat{a}_{h}^{\dagger} \hat{a}_{k}\right) \psi_{k}\right\rangle \geq 0$ to first order in $\tau$, so that the positivity property (3.11) is satisfied.

As a preliminary check of the formalism let us report that the calculation of $\mathcal{L}^{\prime}\left(\hat{a}_{h}^{\dagger} \hat{a}_{k}\right)$ yields the typical structure of the collision term of the Boltzmann equation with the Pauli principle corrections: $\frac{1}{\hbar} \hat{R}_{h \lambda}^{(2) \dagger} \hat{R}_{h \lambda}^{(2)}$ and $-\frac{1}{\hbar}\left[\hat{\Gamma}^{(2)}, \hat{a}_{h}^{\dagger}\right] \hat{a}_{h}+$ c.c. are respectively the "gain" and the "loss" part of the collision term; $\mathcal{L}^{\prime}\left(\hat{a}_{h}^{\dagger} \hat{a}_{k}\right)$ yields also the streaming term of the 
Boltzmann equation. The study of $\mathcal{L}^{\prime}\left(\hat{a}_{h_{1}}^{\dagger} \hat{a}_{h_{2}}^{\dagger} \hat{a}_{k_{2}} \hat{a}_{k_{1}}\right)$ is not yet finished, it should end up with a full hydrodynamic and kinetic description of a one component continuum. The approximations leading to $\mathcal{L}^{\prime}$ are based on the smoothness assumption related to the condition $\tau_{0} \ll \tau_{1}$ and to a "one mode" approximation for the description of the dynamics in the time interval $\tau_{0}$. A finite parameter $\varepsilon \simeq \hbar \tau_{0}$ appears in the formal expression of $\mathcal{L}^{\prime}$; the final results do not appreciably depend on this by-product of the approximations if $\tau_{0} \ll \tau_{1}$. In a sense the simple thermodynamic behaviour expressed by (3.10) arises by an approximation and this is indicated by the presence of $\varepsilon$ : an appreciable dependence of the results on $\varepsilon$ indicates a failure of the smoothness assumption and of the related approximations. Let us stress finally that in this approach existence of closed evolution equations for the thermodynamic state variables avoids any factorisation assumption for the distribution functions and therefore goes far beyond the approach based on the truncation of a hierarchy.

\section{Dynamics with memory effects}

According to $\S 3$, when $\frac{i}{\hbar}[\hat{H}, \cdot]$ can be replaced by the map $\mathcal{L}^{\prime}$ given by (3.19) the family of generalised Gibbs states $\hat{w}(t)$ replaces the family of statistical operators $\hat{\varrho}_{t}=\mathcal{U}\left(t-t_{0}\right) \hat{\varrho}_{t_{0}}$ (for simplicity we assume now time-translation invariance). This means that to determine the evolution of the thermodynamic state from a given time $\bar{t}$ onwards nothing else than $\beta, \mu, \mathbf{v}$ at that time has to be taken into account: i.e. no bias comes by the previous history $\beta\left(\mathbf{x}, t^{\prime}\right), \mu\left(\mathbf{x}, t^{\prime}\right), \mathbf{v}\left(\mathbf{x}, t^{\prime}\right), t^{\prime}<\bar{t}$. This is no longer true if the condition $\tau_{1} \gg \tau_{0}$ [see eq. (3.12)] is not satisfied. Let us assume that at an initial time $\mathrm{T}$ the statistical operator $\hat{\varrho}_{T}$ can be identified with the Gibbs state $\hat{w}(T)$ related to it:

$$
\hat{\varrho}_{T}=\hat{w}(T) .
$$

By a straightforward calculation [18] one has:

$$
\begin{aligned}
\hat{\varrho}_{t} & =e^{-\frac{i}{\hbar} \hat{H}(t-T)} \hat{\varrho}_{T} e^{\frac{i}{\hbar} \hat{H}(t-T)}=\frac{e^{-\left\langle\beta(T) \cdot \hat{e}^{(0)}[-(t-T)]\right\rangle+\left\langle[\mu(T) \beta(T)] \cdot \hat{\rho}_{m}[-(t-T)]\right\rangle}}{\operatorname{Tr} e^{-\left\langle\beta(T) \cdot \hat{e}^{(0)}[-(t-T)]\right\rangle+\left\langle[\mu(T) \beta(T)] \cdot \hat{\rho}_{m}[-(t-T)]\right\rangle}} \\
& =\frac{e^{-\left\langle\beta(t) \cdot \hat{e}^{(0)}\right\rangle+\left\langle[\mu(t) \beta(t)] \cdot \hat{\rho}_{m}\right\rangle-\int_{0}^{t-T} d \tau \frac{d}{d \tau}\left(\left\langle\beta(t-\tau) \cdot \hat{e}^{(0)}(-\tau)\right\rangle-\left\langle[\mu(t-\tau) \beta(t-\tau)] \cdot \hat{\rho}_{m}(-\tau)\right\rangle\right)}}{\operatorname{Tr} e^{-\left\langle\beta(t) \cdot \hat{e}^{(0)}\right\rangle+\left\langle[\mu(t) \beta(t)] \cdot \hat{\rho}_{m}\right\rangle-\int_{0}^{t-T} d \tau \frac{d}{d \tau}\left(\left\langle\beta(t-\tau) \cdot \hat{e}^{(0)}(-\tau)\right\rangle-\left\langle[\mu(t-\tau) \beta(t-\tau)] \cdot \hat{\rho}_{m}(-\tau)\right\rangle\right)}}
\end{aligned}
$$

where $\hat{A}(\tau)=e^{\frac{i}{\hbar} \hat{H} \tau} \hat{A} e^{-\frac{i}{\hbar} \hat{H} \tau}$ and $\langle\beta(t) \cdot \hat{A}\rangle=\int_{\omega} d^{3} \mathbf{x} \beta(t, \mathbf{x}) \hat{A}(\mathbf{x})$. In the last term of the exponent the history of the thermodynamic state during the time interval $[T, t]$ appears; by $\dot{\hat{\rho}}_{m}=-\operatorname{div} \hat{\mathbf{J}}_{m}^{(0)}, \dot{\hat{e}}=-\operatorname{div} \hat{\mathbf{J}}_{l}^{(0)}$ it can be rewritten in the more perspicuous form:

$$
\begin{aligned}
-\int_{0}^{t-T} d \tau \frac{d}{d \tau}\left(\left\langle\beta(t-\tau) \cdot \hat{e}^{(0)}(-\tau)\right\rangle-\left\langle[\mu(t-\tau) \beta(t-\tau)] \cdot \hat{\rho}_{m}(-\tau)\right\rangle\right)= \\
=\int_{T}^{t} d \tau^{\prime}\left[\left\langle\frac{d}{d \tau^{\prime}} \beta\left(\tau^{\prime}\right) \cdot \hat{e}^{(0)}\left(\tau^{\prime}-t\right)\right\rangle-\left\langle\frac{d}{d \tau^{\prime}}\left[\beta\left(\tau^{\prime}\right) \mu\left(\tau^{\prime}\right)\right] \cdot \hat{\rho}_{m}^{(0)}\left(\tau^{\prime}-t\right)\right\rangle\right. \\
\left.\quad-\left\langle\operatorname{grad} \beta\left(\tau^{\prime}\right) \cdot \hat{\mathbf{J}}_{l}^{(0)}\left(\tau^{\prime}-t\right)\right\rangle+\left\langle\operatorname{grad}\left(\beta\left(\tau^{\prime}\right) \mu\left(\tau^{\prime}\right)\right) \cdot \hat{\mathbf{J}}_{m}^{(0)}\left(\tau^{\prime}-t\right)\right\rangle\right] \\
+\int_{T}^{t_{0}} d \tau^{\prime} \int_{\partial \omega} d \sigma \mathbf{n} \cdot\left(\left\langle\beta\left(\tau^{\prime}, \mathbf{x}\right) \hat{\mathbf{J}}_{l}^{(0)}\left(\mathbf{x}, \tau^{\prime}-t\right)\right\rangle-\left\langle\beta\left(\tau^{\prime}, \mathbf{x}\right) \mu\left(\tau^{\prime}, \mathbf{x}\right) \hat{\mathbf{J}}_{m}^{(0)}\left(\mathbf{x}, \tau^{\prime}-t\right)\right\rangle\right)
\end{aligned}
$$


where time and space derivatives of the thermodynamic fields appear on the same footing; by the last term also matter and energy exchanges with the environment during the preparation time $\left[T, t_{o}\right]$ can be described. Taking expression (4.22) for $\hat{\varrho}_{t}$ to calculate the basic expectation values and thus determining the thermodynamic state variables for $t>t_{0}$ as in (3.10), one has for them closed evolution equations having as input $\beta\left(\mathbf{x}, \tau^{\prime}\right), \mu\left(\mathbf{x}, \tau^{\prime}\right), \mathbf{v}\left(\mathbf{x}, \tau^{\prime}\right) \tau^{\prime} \in\left[T, t_{0}\right]$. Such equations are generally used and work under the hypothesis that memory decays within a typical correlation time. This also helps to attenuate the problem of the initial choice (4.21). We mention in passing that no problem about condition (4.21) exists if one assumes the point of view of "informational thermodynamics": then $\hat{\varrho}_{T}=\hat{w}(T)$ is just dictated by the measured values of the relevant variables at time $T$; however this approach does not explain why the previous history of a concrete collection of macrosystems is irrelevant just before $T$. Let us also mention the solution given by Zubarev: $T$ is shifted to $-\infty$, thus taking off any previous history; however this limit is highly critical and since also a thermodynamic limit is involved, it shifts the problem of thermodynamic evolution to a cosmological one. In our framework a time scale is associated to the system and the generator $\mathcal{L}^{\prime}$ should have a non-Hamiltonian part. This provides a mechanism by which memory can decay. Let us assume that the preparation time $t_{o}-T$ is larger than the decay time of the memory: at this point the history that comes before $T$ is irrelevant for the dynamics that $\mathcal{L}^{\prime}$ is able to describe. Then the choice (4.21), that is not biased by this history, is adequate. When $\mathcal{L}^{\prime}=\frac{i}{\hbar}[\hat{H}, \cdot]+\tilde{\mathcal{L}}^{\prime}$, calling $\hat{\varrho}([\beta, \mu, \mathbf{v}], t, T)$ the operator on the l.h.s. of (4.22) one has:

$$
\begin{aligned}
& \operatorname{Tr}\left(\hat{A} \mathcal{U}(t, T) \hat{\varrho}_{T}\right)=\operatorname{Tr}\left[\left(T e^{\int_{T}^{t} d \tau \tilde{\mathcal{L}}^{\prime}(\tau)} \hat{A}\right) \hat{\varrho}([\beta, \mu, \mathbf{v}], t, T)\right], \\
& \tilde{\mathcal{L}}^{\prime}(\tau)=e^{\mathcal{L}_{0}{ }^{\prime}(T-\tau)} \tilde{\mathcal{L}}^{\prime} e^{-\mathcal{L}_{0}{ }^{\prime}(T-\tau)}, \quad \mathcal{L}_{0}{ }^{\prime}=\frac{i}{\hbar}[\hat{H}, \cdot] .
\end{aligned}
$$

\section{References}

[1] G. C. Ghirardi, P. Pearle and A. Rimini, Phys. Rev. A 42, 78 (1990).

[2] G. Ludwig, Foundations of Quantum Mechanics (1983, Springer, Berlin).

[3] E. B. Davies, Quantum theory of open systems (1976, Academic Press, London).

[4] G. Lindblad, Commun. Math. Phys. 48, 119 (1976).

[5] E. B. Davies, Commun. Math. Phys. 15, 227 (1969); 19, 83 (1970); 22, 51 (1971). A. Barchielli, L. Lanz, G. M. Prosperi, Nuovo Cimento 72B, 79 (1982); Found. Phys. 13, 779 (1983); Proceedings of the International Symposium: Foundations of Quantum Mechanics in the Light of New Technology (Tokyo, 1983), p.165; A. Barchielli, G. Lupieri, J. Math. Phys. 26, 2222 (1985); A. S. Holevo, in Lect. Notes in Mathematics (1988, Springer, Berlin), vol. 1303, p.128; Lect. Notes in Mathematics (1989, Springer, Berlin), vol. 1396, p.229; L. Lanz and O. Melsheimer, in Quantum Mechanics and Trajectories - Symposium On the Foundations of Modern Physics, edited by P. Busch, P. J. Lahti and P. Mittelstaedt (World Scientific, 1993) p.233-241; 
V. P. Belavkin, in A. Blaquière (ed.), Modelling and Control of Systems, Lect. Notes in Control and Information Sciences, (1988, Springer, Berlin), vol. 121, p.245; V. P. Belavkin, Phys. Lett. 140A, 355 (1989); V. P. Belavkin and P. Staszewski, Phys. Lett. 140A, 359 (1989).

[6] L. Lanz, Int. J. Theor. Phys. 33, 19 (1994).

[7] L. Lanz and O. Melsheimer, Nuovo Cimento 108B, 511 (1993).

[8] L. Lanz and B. Vacchini, Int. J. Theor. Phys. 36, 67 (1997).

[9] H. Rauch, in Advances in Quantum Phenomena, edited by E. G. Beltrametti and J.-M. Lévy-Leblond, NATO ASI series, Vol. B347, (1995, Plenum Press, New York) p.113.

[10] V. F. Sears, Neutron Optics (1989, Oxford University Press, Oxford).

[11] C. S. Adams, M. Siegel, J. Mlynek, Phys. Rep. 240, 143 (1994).

[12] J. Vigué, Phys. Rev. A 52, 3973 (1995).

[13] M. D. Srinivas and E. B. Davies, Optica Acta 28, 981 (1981).

[14] L. Diósi, Europhys. Lett. 30, 63 (1995).

[15] L. Lanz, O. Melsheimer and E. Wacker, Physica 131A, 520 (1985).

[16] A. S. Holevo, Probabilistic and Statistical Aspects of Quantum Theory (1982, North Holland, Amsterdam).

[17] V. G. Morozov and G. Roepke, Physica 221A, 511 (1995).

[18] W. A. Robin, J. Phys. A 23, 2065 (1990).

[19] D. N. Zubarev, Non-equilibrium statistical thermodynamics, (1974, Consultant Bureau, New York). 